\title{
Lyapunov-type inequalities for a class of fractional differential equations
}

\section{Donal O'Regan ${ }^{1,2}$ and Bessem Samet ${ }^{3 *}$}

"Correspondence:

bsamet@ksu.edu.sa

${ }^{3}$ Department of Mathematics,

College of Science, King Saud

University, P.O. Box 2455, Riyadh,

11451, Saudi Arabia

Full list of author information is

available at the end of the article

\section{Abstract}

In this paper, we establish new Lyapunov-type inequalities for a class of fractional boundary value problems. As an application, we obtain a lower bound for the eigenvalues of corresponding equations.

MSC: 26D10; 34A08; 34B09

Keywords: Lyapunov's inequality; fractional boundary value problem; Green's function; eigenvalue

\section{Introduction}

Let $u$ be a nontrivial solution to the second order differential equation

$$
u^{\prime \prime}(t)+q(t) u(t)=0, \quad a<t<b
$$

with the Dirichlet boundary condition

$$
u(a)=u(b)=0,
$$

where $q:[a, b] \rightarrow \mathbb{R}$ is continuous. Then the so-called Lyapunov inequality [1]

$$
(b-a) \int_{a}^{b}|q(s)| d s>4
$$

holds, and constant 4 in (1.3) cannot be replaced by a larger number. The above inequality has several applications to various problems related to differential equations.

There are several generalizations and extensions of Lyapunov's result. Hartman and Wintner [2] proved that if $u$ is a nontrivial solution to (1.1)-(1.2), then

$$
\int_{a}^{b}(b-s)(s-a) q^{+}(s) d s>b-a,
$$

where $q^{+}(s)$ is the positive part of $q$, defined as

$$
q^{+}(s)=\max \{q(s), 0\} .
$$

(c) 2015 O'Regan and Samet. This article is distributed under the terms of the Creative Commons Attribution 4.0 International License (http://creativecommons.org/licenses/by/4.0/), which permits unrestricted use, distribution, and reproduction in any medium, provided you give appropriate credit to the original author(s) and the source, provide a link to the Creative Commons license, and indicate if changes were made. 
For other generalizations and extensions of the classical Lyapunov's inequality, we refer to [2-17] and the references therein.

Recently, some Lyapunov-type inequalities for fractional boundary value problems have been obtained. In [9], Ferreira established a Lyapunov-type inequality for a differential equation that depends on the Riemann-Liouville fractional derivative, i.e., for the boundary value problem

$$
\begin{aligned}
& \left({ }_{a} D^{\alpha} u\right)(t)+q(t) u(t)=0, \quad a<t<b, 1<\alpha \leq 2, \\
& u(a)=u(b)=0,
\end{aligned}
$$

where he proved that if $u$ is a nontrivial continuous solution to the above problem, then

$$
\int_{a}^{b}|q(s)| d s>\frac{\Gamma(\alpha) \alpha^{\alpha}}{[(\alpha-1)(b-a)]^{\alpha-1}}
$$

In [8], Ferreira obtained a Lyapunov-type inequality for the Caputo fractional boundary value problem

$$
\begin{aligned}
& \left({ }_{a}^{C} D^{\alpha} u\right)(t)+q(t) u(t)=0, \quad a<t<b, 1<\alpha \leq 2, \\
& u(a)=u(b)=0,
\end{aligned}
$$

where he established that if $u$ is a nontrivial continuous solution to the above problem, then

$$
\int_{a}^{b}|q(s)| d s>\Gamma(\alpha)\left(\frac{4}{b-a}\right)^{\alpha-1}
$$

Observe that if we set $\alpha=2$ in (1.4) or (1.5), one can obtain the classical Lyapunov inequality (1.3). In [11], Jleli and Samet studied the fractional differential equation

$$
\left({ }_{a}^{C} D^{\alpha} u\right)(t)+q(t) u(t)=0, \quad a<t<b, 1<\alpha \leq 2
$$

with mixed boundary conditions

$$
u(a)=u^{\prime}(b)=0
$$

or

$$
u^{\prime}(a)=u(b)=0
$$

For boundary conditions (1.6) and (1.7), two Lyapunov-type inequalities were established respectively as follows:

$$
\int_{a}^{b}(b-s)^{\alpha-2}|q(s)| d s \geq \frac{\Gamma(\alpha)}{\max \{\alpha-1,2-\alpha\}(b-a)}
$$


and

$$
\int_{a}^{b}(b-s)^{\alpha-1}|q(s)| d s \geq \Gamma(\alpha)
$$

Rong and Bai [16] established a Lyapunov-type inequality for the above fractional differential equation with the fractional boundary conditions

$$
{ }_{a}^{C} D^{\beta} u(b)=u(a)=0
$$

where $0<\beta \leq 1$ and $1<\alpha \leq \beta+1$. They established the following result: if a nontrivial continuous solution to the above fractional boundary value problem exists, then

$$
\int_{a}^{b}(b-s)^{\alpha-\beta-1}|q(s)| d s \geq \frac{(b-a)^{-\beta}}{\max \left\{\frac{1}{\Gamma(\alpha)}-\frac{\Gamma(2-\beta)}{\Gamma(\alpha-\beta)}, \frac{\Gamma(2-\beta)}{\Gamma(\alpha-\beta)},\left(\frac{2-\alpha}{\alpha-1}\right) \frac{\Gamma(2-\beta)}{\Gamma(\alpha-\beta)}\right\}} .
$$

Observe that if $\beta=1$, then (1.9) reduces to the Lyapunov-type inequality (1.8). For other related works, we refer to [18-21].

In all the above cited works, the fractional order $\alpha$ belongs to (1.2]. In this paper, we are concerned with the problem of finding new Lyapunov-type inequalities for the fractional boundary value problem

$$
\begin{aligned}
& \left({ }_{a} D^{\alpha} u\right)(t)+q(t) u(t)=0, \quad a<t<b, 3<\alpha \leq 4, \\
& u(a)=u^{\prime}(a)=u^{\prime \prime}(a)=u^{\prime \prime}(b)=0,
\end{aligned}
$$

where ${ }_{a} D^{\alpha}$ is the standard Riemann-Liouville fractional derivative of fractional order $\alpha$ and $q:[a, b] \rightarrow \mathbb{R}$ is a continuous function. As an application, we obtain a lower bound for the eigenvalues of the corresponding problem.

Let $f$ be a real function defined on $[a, b](a<b)$.

Definition 1.1 The integral

$$
\left({ }_{a} I^{\alpha} f\right)(t)=\frac{1}{\Gamma(\alpha)} \int_{a}^{t}(t-s)^{\alpha-1} f(s) d s, \quad t \in[a, b]
$$

where $\alpha>0$, is called the Riemann-Liouville fractional integral of order $\alpha$, and $\Gamma(\alpha)$ is the Euler gamma function defined by

$$
\Gamma(\alpha)=\int_{0}^{\infty} t^{\alpha-1} e^{-t} d t, \quad \alpha>0
$$

Definition 1.2 The expression

$$
{ }_{a} D^{\alpha} f(t)=\frac{1}{\Gamma(n-\alpha)}\left(\frac{d}{d t}\right)^{n} \int_{a}^{t} \frac{f(s)}{(t-s)^{\alpha-n+1}} d s,
$$

where $n=[\alpha]+1,[\alpha]$ denotes the integer part of number $\alpha$, is called the Riemann-Liouville fractional derivative of order $\alpha$. 
The following lemma is crucial in finding an integral representation of the fractional boundary value problem (1.10)-(1.11).

Lemma 1.3 Assume that $f \in C(a, b) \cap L(a, b)$ with a fractional derivative of order $\alpha>0$ that belongs to $C(a, b) \cap L(a, b)$. Then

$$
{ }_{a} I^{\alpha}{ }_{a} D^{\alpha} f(t)=f(t)+c_{1}(t-a)^{\alpha-1}+c_{2}(t-a)^{\alpha-2}+\cdots+c_{n}(t-a)^{\alpha-n},
$$

for some constants $c_{i} \in \mathbb{R}, i=1, \ldots, n, n=[\alpha]+1$.

For more details on fractional calculus, we refer the reader to [22-24].

\section{Main results}

The following lemmas will be needed.

Lemma 2.1 We have that $u \in C[a, b]$ is a solution to the boundary value problem (1.10)(1.11) if and only if $u$ satisfies the integral equation

$$
u(t)=\int_{a}^{b} G(t, s) q(s) u(s) d s
$$

where $G(t, s)$ is the Green function of problem (1.10)-(1.11) defined as

$$
G(t, s)=\frac{1}{\Gamma(\alpha)} \begin{cases}\frac{(t-a)^{\alpha-1}(b-s)^{\alpha-3}}{(b-a)^{\alpha-3}}-(t-s)^{\alpha-1}, & a \leq s \leq t \leq b, \\ \frac{(t-a)^{\alpha-1}(b-s)^{\alpha-3}}{(b-a)^{\alpha-3}}, & a \leq t \leq s \leq b .\end{cases}
$$

Proof From Lemma 1.3, $u \in C[a, b]$ is a solution to the boundary value problem (1.10)(1.11) if and only if

$$
u(t)=c_{1}(t-a)^{\alpha-1}+c_{2}(t-a)^{\alpha-2}+c_{3}(t-a)^{\alpha-3}+c_{4}(t-a)^{\alpha-4}-\frac{1}{\Gamma(\alpha)} \int_{a}^{t}(t-s)^{\alpha-1} q(s) u(s) d s
$$

for some real constants $c_{i}, i=1, \ldots, 4$. Using the boundary conditions $u(a)=u^{\prime}(a)=u^{\prime \prime}(a)=$ 0 , we get immediately

$$
c_{2}=c_{3}=c_{4}=0 \text {. }
$$

The boundary condition $u^{\prime \prime}(b)=0$ yields

$$
c_{1}=\frac{1}{(b-a)^{\alpha-3} \Gamma(\alpha)} \int_{a}^{b}(b-s)^{\alpha-3} q(s) u(s) d s .
$$

Hence

$$
u(t)=\frac{(t-a)^{\alpha-1}}{(b-a)^{\alpha-3} \Gamma(\alpha)} \int_{a}^{b}(b-s)^{\alpha-3} q(s) u(s) d s-\frac{1}{\Gamma(\alpha)} \int_{a}^{t}(t-s)^{\alpha-1} q(s) u(s) d s,
$$

which concludes the proof. 
Lemma 2.2 The function G defined in Lemma 2.1 satisfies the following property:

$$
0 \leq G(t, s) \leq G(b, s)=\frac{(b-s)^{\alpha-3}(s-a)(2 b-a-s)}{\Gamma(\alpha)}, \quad(t, s) \in[a, b] \times[a, b] .
$$

Proof We start by fixing an arbitrary $s \in(a, b]$. Differentiating $G(t, s)$ with respect to $t$, we get

$$
\partial_{t} G(t, s)=\frac{(\alpha-1)}{\Gamma(\alpha)} \begin{cases}\frac{(t-a)^{\alpha-2}(b-s)^{\alpha-3}}{(b-a))^{\alpha-3}}-(t-s)^{\alpha-2}, & a \leq s \leq t \leq b, \\ \frac{(t-a)^{\alpha-2}(b-s)^{\alpha-3}}{(b-a)^{\alpha-3}}, & a \leq t \leq s \leq b .\end{cases}
$$

For $a \leq t \leq s \leq b$, we have

$$
\frac{\Gamma(\alpha)}{(\alpha-1)} \partial_{t} G(t, s)=\frac{(t-a)^{\alpha-2}(b-s)^{\alpha-3}}{(b-a)^{\alpha-3}} \geq 0,
$$

while for $a \leq s \leq t \leq b$, we have

$$
\begin{aligned}
\frac{\Gamma(\alpha)}{(\alpha-1)} \partial_{t} G(t, s) & =\frac{(t-a)^{\alpha-2}(b-s)^{\alpha-3}}{(b-a)^{\alpha-3}}-(t-s)^{\alpha-2} \\
& =\frac{(t-a)^{\alpha-2}((b-a)-(s-a))^{\alpha-3}}{(b-a)^{\alpha-3}}-((t-a)-(s-a))^{\alpha-2} \\
& =(t-a)^{\alpha-2}\left(1-\frac{s-a}{b-a}\right)^{\alpha-3}-(t-a)^{\alpha-2}\left(1-\frac{s-a}{t-a}\right)^{\alpha-2} \\
& \geq(t-a)^{\alpha-2}\left(1-\frac{s-a}{b-a}\right)^{\alpha-3}-(t-a)^{\alpha-2}\left(1-\frac{s-a}{b-a}\right)^{\alpha-2} \\
& =(t-a)^{\alpha-2}\left[\left(1-\frac{s-a}{b-a}\right)^{\alpha-3}-\left(1-\frac{s-a}{b-a}\right)^{\alpha-2}\right] \\
& \geq 0 .
\end{aligned}
$$

Consequently, the function $G(t, s)$ is non-decreasing with respect to $t$, from which it follows that

$$
0=G(a, s) \leq G(t, s) \leq G(b, s), \quad(t, s) \in[a, b] \times[a, b] .
$$

The proof is complete.

We have the following Hartman-Wintner-type inequality.

Theorem 2.3 If a nontrivial continuous solution to the fractional boundary value problem

$$
\begin{aligned}
& \left({ }_{a} D^{\alpha} u\right)(t)+q(t) u(t)=0, \quad a<t<b, 3<\alpha \leq 4, \\
& u(a)=u^{\prime}(a)=u^{\prime \prime}(a)=u^{\prime \prime}(b)=0
\end{aligned}
$$

exists, where $q$ is a real and continuous function in $[a, b]$, then

$$
\int_{a}^{b}(b-s)^{\alpha-3}(s-a)(2 b-a-s)|q(s)| d s \geq \Gamma(\alpha) .
$$


Proof Let $\mathcal{B}=C[a, b]$ be the Banach space endowed with the norm

$$
\|y\|_{\infty}=\max _{a \leq t \leq b}|y(t)|, \quad y \in \mathcal{B} .
$$

It follows from Lemma 2.1 that a solution $u$ to (1.10)-(1.11) satisfies the integral equation

$$
u(t)=\int_{a}^{b} G(t, s) q(s) u(s) d s, \quad t \in[a, b] .
$$

Thus, for all $t \in[a, b]$, we have

$$
\begin{aligned}
|u(t)| & \leq \int_{a}^{b}|G(t, s)||q(s)||u(s)| d s \\
& \leq\left(\int_{a}^{b} \sup _{a \leq t \leq b}|G(t, s) \| q(s)| d s\right)\|u\|_{\infty},
\end{aligned}
$$

which yields

$$
\|u\|_{\infty} \leq\left(\int_{a}^{b} \sup _{a \leq t \leq b}|G(t, s) \| q(s)| d s\right)\|u\|_{\infty}
$$

Since $u$ is nontrivial, then $\|u\|_{\infty} \neq 0$, so

$$
1 \leq \int_{a}^{b} \sup _{a \leq t \leq b}|G(t, s)||q(s)| d s .
$$

Now, an application of Lemma 2.2 yields

$$
1 \leq \int_{a}^{b} G(b, s)|q(s)| d s
$$

from which the inequality in (2.1) follows.

Corollary 2.4 If a nontrivial continuous solution to the fractional boundary value problem

$$
\begin{aligned}
& \left({ }_{a} D^{\alpha} u\right)(t)+q(t) u(t)=0, \quad a<t<b, 3<\alpha \leq 4, \\
& u(a)=u^{\prime}(a)=u^{\prime \prime}(a)=u^{\prime \prime}(b)=0
\end{aligned}
$$

exists, where $q$ is a real and continuous function in $[a, b]$, then

$$
\int_{a}^{b}(b-s)^{\alpha-3}(s-a)|q(s)| d s \geq \frac{\Gamma(\alpha)}{2(b-a)}
$$

Proof From Theorem 2.3, we have

$$
\int_{a}^{b}(b-s)^{\alpha-3}(s-a)(2 b-a-s)|q(s)| d s \geq \Gamma(\alpha) .
$$


Next we note

$$
2 b-a-s \leq 2(b-a), \quad s \in[a, b] .
$$

Thus we get

$$
2(b-a) \int_{a}^{b}(b-s)^{\alpha-3}(s-a)|q(s)| d s \geq \Gamma(\alpha),
$$

which gives the desired inequality (2.2).

We have the following Lyapunov-type inequality.

Corollary 2.5 If a nontrivial continuous solution to the fractional boundary value problem

$$
\begin{aligned}
& \left({ }_{a} D^{\alpha} u\right)(t)+q(t) u(t)=0, \quad a<t<b, 3<\alpha \leq 4, \\
& u(a)=u^{\prime}(a)=u^{\prime \prime}(a)=u^{\prime \prime}(b)=0
\end{aligned}
$$

exists, where $q$ is a real and continuous function in $[a, b]$, then

$$
\int_{a}^{b}|q(s)| d s \geq \frac{\Gamma(\alpha)(\alpha-2)^{\alpha-2}}{2(\alpha-3)^{\alpha-3}(b-a)^{\alpha-1}} .
$$

Proof Let

$$
\psi(s)=(b-s)^{\alpha-3}(s-a), \quad s \in[a, b] .
$$

Now, we differentiate $\psi(s)$ on $(a, b)$, and we obtain after simplifications

$$
\psi^{\prime}(s)=(b-s)^{\alpha-4}[(b-s)-(\alpha-3)(s-a)] .
$$

Observe that $\psi^{\prime}(s)$ has a unique zero, attained at the point

$$
s^{*}=\frac{b+(\alpha-3) a}{\alpha-2} .
$$

It is easily seen that $s^{*} \in(a, b), \psi^{\prime}(s)>0$ on $\left(a, s^{*}\right)$, and $\psi^{\prime}(s)<0$ on $\left(s^{*}, b\right)$. We conclude that

$$
\max _{a \leq s \leq b} \psi(s)=\psi\left(s^{*}\right)=(\alpha-3)^{\alpha-3}\left(\frac{b-a}{\alpha-2}\right)^{\alpha-2} .
$$

From Corollary 2.4, we have

$$
\int_{a}^{b} \psi(s)|q(s)| d s \geq \frac{\Gamma(\alpha)}{2(b-a)}
$$

which yields

$$
\int_{a}^{b}|q(s)| d s \geq \frac{\Gamma(\alpha)}{2(b-a) \psi\left(s^{*}\right)}
$$


from which inequality (2.3) follows.

Corollary 2.6 If a nontrivial continuous solution to the boundary value problem

$$
\begin{aligned}
& u^{\prime \prime \prime \prime}(t)+q(t) u(t)=0, \quad a<t<b, \\
& u(a)=u^{\prime}(a)=u^{\prime \prime}(a)=u^{\prime \prime}(b)=0
\end{aligned}
$$

exists, where $q$ is a real and continuous function in $[a, b]$, then

$$
\int_{a}^{b}(b-s)(s-a)(2 b-a-s)|q(s)| d s \geq 6 .
$$

Proof Inequality (2.4) follows from Theorem 2.3 with $\alpha=4$.

Corollary 2.7 If a nontrivial continuous solution to the boundary value problem

$$
\begin{aligned}
& u^{\prime \prime \prime \prime}(t)+q(t) u(t)=0, \quad a<t<b, \\
& u(a)=u^{\prime}(a)=u^{\prime \prime}(a)=u^{\prime \prime}(b)=0
\end{aligned}
$$

exists, where $q$ is a real and continuous function in $[a, b]$, then

$$
\int_{a}^{b}(b-s)(s-a)|q(s)| d s \geq \frac{3}{b-a} .
$$

Proof Inequality (2.5) follows from Corollary 2.4 with $\alpha=4$.

Corollary 2.8 If a nontrivial continuous solution to the boundary value problem

$$
\begin{aligned}
& u^{\prime \prime \prime \prime}(t)+q(t) u(t)=0, \quad a<t<b, \\
& u(a)=u^{\prime}(a)=u^{\prime \prime}(a)=u^{\prime \prime}(b)=0
\end{aligned}
$$

exists, where $q$ is a real and continuous function in $[a, b]$, then

$$
\int_{a}^{b}|q(s)| d s \geq \frac{12}{(b-a)^{3}} .
$$

Proof Inequality (2.6) follows from Corollary 2.5 with $\alpha=4$.

\section{Application}

In this section, we give an application of the Hartman-Wintner-type inequality (2.2) for the eigenvalue problem

$$
\begin{aligned}
& \left({ }_{0} D^{\alpha} u\right)(t)+\lambda u(t)=0, \quad 0<t<1,3<\alpha \leq 4, \\
& u(0)=u^{\prime}(0)=u^{\prime \prime}(0)=u^{\prime \prime}(1)=0 .
\end{aligned}
$$


Theorem 3.1 If $\lambda$ is an eigenvalue to the fractional boundary value problem (3.1)-(3.2), then

$$
|\lambda| \geq \frac{\Gamma(\alpha)}{2 B(2, \alpha-2)},
$$

where $B$ is the beta function defined by

$$
B(x, y)=\int_{0}^{1} s^{x-1}(1-s)^{y-1} d s, \quad x, y>0 .
$$

Proof Let $\lambda$ be an eigenvalue to (3.1)-(3.2). Then there exists $u=u_{\lambda}$, a nontrivial solution to (3.1)-(3.2). An application of Corollary 2.4 yields

$$
|\lambda| \int_{0}^{1}(1-s)^{\alpha-3} s d s \geq \frac{\Gamma(\alpha)}{2} .
$$

Now,

$$
\int_{0}^{1}(1-s)^{\alpha-3} s d s=\int_{0}^{1} s^{2-1}(1-s)^{(\alpha-2)-1} d s=B(2, \alpha-2),
$$

from which we obtain

$$
|\lambda| B(2, \alpha-2) \geq \frac{\Gamma(\alpha)}{2} .
$$

The proof is complete.

\section{Competing interests}

The authors declare that they have no competing interests.

\section{Authors' contributions}

All authors contributed equally in drafting this manuscript and giving the main proofs. All authors read and approved the final manuscript.

\section{Author details}

${ }^{1}$ School of Mathematics, Statistics and Applied Mathematics, National University of Ireland, Galway, Ireland. ${ }^{2}$ Nonlinear Analysis and Applied Mathematics (NAAM)-Research Group, Department of Mathematics, King Abdulaziz University, Jeddah, Saudi Arabia. ${ }^{3}$ Department of Mathematics, College of Science, King Saud University, P.O. Box 2455, Riyadh, 11451, Saudi Arabia.

\section{Acknowledgements}

The second author would like to extend his sincere appreciation to the Deanship of Scientific Research at King Saud University for its funding of this research through the International Research Group Project No. IRG14-04.

Received: 30 March 2015 Accepted: 24 July 2015 Published online: 08 August 2015

\section{References}

1. Lyapunov, AM: Problème général de la stabilité du mouvement. Ann. of Math. Stud., vol. 17. Princeton University Press, Princeton (1949)

2. Hartman, P, Wintner, A: On an oscillation criterion of Liapunov. Am. J. Math. 73, 885-890 (1951)

3. Aktas, MF: Lyapunov-type inequalities for a certain class of $n$-dimensional quasilinear systems. Electron. J. Differ. Equ. 2013, 67 (2013)

4. Çakmak, D: On Lyapunov-type inequality for a class of nonlinear systems. Math. Inequal. Appl. 16, 101-108 (2013)

5. Çakmak, D: Lyapunov-type integral inequalities for certain higher order differential equations. Appl. Math. Comput. 216, 368-373 (2010)

6. Cheng, SS: A discrete analogue of the inequality of Lyapunov. Hokkaido Math. J. 12, 105-112 (1983)

7. Eliason, SB: A Lyapunov inequality for a certain nonlinear differential equation. J. Lond. Math. Soc. 2, 461-466 (1970)

8. Ferreira, RAC: A Lyapunov-type inequality for a fractional boundary value problem. Fract. Calc. Appl. Anal. 16(4), 978-984 (2013) 
9. Ferreira, RAC: On a Lyapunov-type inequality and the zeros of a certain Mittag-Leffler function. J. Math. Anal. Appl. 412, 1058-1063 (2014)

10. He, X, Tang, XH: Lyapunov-type inequalities for even order differential equations. Commun. Pure Appl. Anal. 11, 465-473 (2012)

11. Jleli, M, Samet, B: Lyapunov-type inequalities for a fractional differential equation with mixed boundary conditions. Math. Inequal. Appl. 18(2), 443-451 (2015)

12. Kwong, MK: On Lyapunov's inequality for disfocality. J. Math. Anal. Appl. 83, 486-494 (1981)

13. Pachpatte, BG: On Lyapunov-type inequalities for certain higher order differential equations. J. Math. Anal. Appl. 195, 527-536 (1995)

14. Pachpatte, BG: Lyapunov type integral inequalities for certain differential equations. Georgian Math. J. 4, 139-148 (1997)

15. Parhi, N, Panigrahi, S: On Lyapunov-type inequality for third-order differential equations. J. Math. Anal. Appl. 233, 445-460 (1999)

16. Rong, J, Bai, C: Lyapunov-type inequality for a fractional differential equation with fractional boundary conditions. Adv. Differ. Equ. 2015, 82 (2015)

17. Yang, X: On Lyapunov-type inequality for certain higher-order differential equations. Appl. Math. Comput. 134, 307-317 (2003)

18. Baleanu, D, Agarwal, P: Certain inequalities involving the fractional $q$-integral operators. Abstr. Appl. Anal. 2014 Article ID 371274 (2014)

19. Choi, J, Agarwal, P: Certain fractional integral inequalities involving hypergeometric operators. East Asian Math. J. 30, 283-291 (2014)

20. Wang, G, Agarwal, P, Baleanu, D: Certain new Gruss type inequalities involving Saigo fractional $q$-integral operator. J. Comput. Anal. Appl. 19(5), 862-873 (2015)

21. Wang, G, Agarwal, $P$, Chand, M: Certain Gruss type inequalities involving the generalized fractional integral operator. J. Inequal. Appl. 2014, 147 (2014)

22. Agarwal, RP, O'Regan, D, Stanek, S: Positive solutions for Dirichlet problems of singular nonlinear fractional differential equations. J. Math. Anal. Appl. 371(1), 57-68 (2010)

23. Hernández, E, O'Regan, D, Balachandran, K: On recent developments in the theory of abstract differential equations with fractional derivatives. Nonlinear Anal. 73(10), 3462-3471 (2010)

24. Kilbas, AA, Srivastava, HM, Trujillo, Jj: Theory and Applications of Fractional Differential Equations. North-Holland Mathematics Studies, vol. 204. Elsevier, Amsterdam (2006)

\section{Submit your manuscript to a SpringerOpen ${ }^{\circ}$ journal and benefit from:}

- Convenient online submission

Rigorous peer review

- Immediate publication on acceptance

- Open access: articles freely available online

- High visibility within the field

- Retaining the copyright to your article 